\title{
ESTRÉS PERCIBIDO E INTENCIÓN DE MIGRAR AL INTERIOR DEL PAIIS EN MÉDICOS Y ENFERMEROS QUE RESIDEN EN LIMA: UN ANÁLISIS EXPLORATORIO DE LA ENCUESTA NACIONAL DE SATISFACCIÓN DE USUARIOS EN SALUD (ENSUSALUD), 2015
}

\author{
Brendy Santiago-Ullero,,a, Sergio Valer-Villanueva1,a, Diego Urrunaga-Pastor,a, Vicente A. Benites-Zapata ${ }^{2, b}$
}

RESUMEN

Objetivos. Identificar la frecuencia de intención de migrar al interior del país y valorar la asociación entre el estrés percibido (EP) y la intención de migrar al interior del país (IMIP) en médicos y enfermeros que residían en Lima Metropolitana durante el 2015. Materiales y métodos. Análisis secundario de datos, empleando la Encuesta Nacional de Satisfacción de Usuarios en Salud (ENSUSALUD) del año 2015. Se incluyeron únicamente a aquellos profesionales que residían en Lima Metropolitana. Se definió como IMIP a aquellos que manifestaron su intención de trabajar en algún departamento diferente de Lima. Para medir el EP se empleó la Perceived stress scale de 14 preguntas, validada en español. Se empleó regresión logística para valorar la asociación planteada. La medida de asociación reportada fue el odds ratio (OR), con su intervalo de confianza al 95\% (IC95\%). Resultados. Se analizaron datos de 796 profesionales, de los cuales el $54,8 \%$ fueron enfermeros. La edad promedio fue de 45,2 años. El 4,9\% (37) de los participantes tuvieron IMIP, de los cuales, el 64,9\% (24) fueron enfermeros. En el análisis de regresión logística multivariado, se encontró asociación entre una mayor percepción de estrés con la IMIP (OR=1,31; IC95\%:1,07-1,60). Conclusiones. La IMIP se presentó en uno de cada 20 profesionales y se asoció a un mayor EP en el personal de la salud estudiado.

Palabras clave: Médicos, Enfermeros; Migración interna; Estrés psicológico; Asignación de recursos para la atención de salud; Perú. (Fuente: DeCS BIREME).

\section{PERCEIVED STRESS AND INTENTION TO MIGRATE TO THE INTERIOR OF PERU AMONG PHYSICIANS AND NURSES WHO RESIDE IN LIMA: EXPLORATORY ANALYSIS OF THE NATIONAL SURVEY OF SATISFACTION OF USERS IN HEALTH, 2015}

\begin{abstract}
Objectives. To identify the frequency of intention to migrate to the interior of Peru (IMIP) and assess the association between perceived stress (PS) and IMIP among physicians and nurses residing in metropolitan Lima in 2015. Materials and methods. This was a secondary analysis of data obtained from the National Survey of Satisfaction of Users in Health (Encuesta Nacional de Satisfacción de Usuarios en Salud-ENSUSALUD) in 2015. Only professionals residing in Metropolitan Lima were included for the analysis. IMIP was defined as the intention to work in a geographical region other than Lima. PS was measured using the 14-question perceived stress scale validated in Spanish. Logistic regression was used to evaluate this association; the measure of association was the odds ratio (OR) with the $95 \%$ confidence interval $(95 \% \mathrm{Cl})$. Results. Data from 796 professionals were analyzed, $54.8 \%$ of whom were nurses. The average age was 45.2 years; $4.9 \%$ (37) of the participants had IMIP, $64.9 \%(24)$ of whom were nurses. The results of multivariate logistic regression analysis indicated an association between higher PS and IMIP (OR: 1.31, 95\% Cl: 1.07-1.60). Conclusions. The IMIP was reported by one of every 20 professionals and was associated with higher PS in the study group.
\end{abstract}

Key words: Physicians; Nurses; Internal migration; Stress, psychological; Health care rationing; Peru. (Source: MeSH NLM).

\footnotetext{
Sociedad Científica de Estudiantes de Medicina de la Universidad de San Martín de Porres, Facultad de Medicina Humana, Universidad de San Martín de Porres. Lima, Perú.

Centro de Investigación en Epidemiología Clínica y Medicina Basada en Evidencias, Instituto de Investigación, Facultad de Medicina Humana, Universidad de San Martín de Porres. Lima, Perú.

a Estudiante de Medicina; ${ }^{\mathrm{b}}$ médico, magíster en Ciencias en Investigación Epidemiológica Recibido: 15/04/2017 Aprobado: 20/09/2017 En línea: 29/09/2017
}

Citar como: Santiago-Ullero B, Valer-Villanueva S, Urrunaga-Pastor D, Benites-Zapata VA. Estrés percibido e intención de migrar al interior del país en médicos y enfermeros que residen en Lima: un análisis exploratorio de la Encuesta Nacional de Satisfacción de Usuarios en Salud (ENSUSALUD), 2015. Rev Peru Med Exp Salud Publica. 2017;34(3): 404-13. doi: 10.17843/rpmesp.2017.343.2822 


\section{INTRODUCCIÓN}

Según la Organización Mundial de la Salud (OMS) (1), en Perú durante el 2013, 18 de sus 25 departamentos tenían, al menos, 25 trabajadores de salud (médicos, enfermeras y obstetras) por cada 10000 habitantes, teniendo Lima, la cifra más alta con 36 trabajadores por cada 10000 habitantes. Además, se apreció una limitación de recursos humanos (RR.HH.) en los departamentos de Loreto (5,1 médicos y 7,7 enfermeras por cada 10000 habitantes), Cajamarca (5,5 médicos y 10,1 enfermeras por cada 10000 habitantes) y San Martín (6,3 médicos y 6,3 enfermeras por cada 10000 habitantes) ${ }^{(1)}$. Coincidentemente, estos departamentos poseen los mayores índices de pobreza en Perú (2). A pesar de la implementación de intervenciones con el objetivo de cubrir el déficit de RR.HH., como bonos monetarios y el servicio rural obligatorio, aún existe una gran brecha de servicios de salud entre las zona rurales y las urbanas ${ }^{(3)}$.

Lima Metropolitana concentra el mayor número de médicos egresados, escuelas de Medicina y establecimientos de salud (EE.SS.), lo que ocasiona una sobreoferta de médicos, lo cual se incrementará en los próximos años ${ }^{(4,5)}$. Adicionalmente, muchos médicos y profesionales de enfermería peruanos migran al extranjero buscando mejores condiciones económicas ${ }^{(6,7)}$. Se ha descrito que uno de cada 20 médicos recién colegiados de Lima refiere su intención de trabajar en el interior del país ${ }^{(8)}$. En cambio, no se registran estudios locales sobre la intención de migrar al interior del país (IMIP) en el personal de enfermería.

El personal sanitario es un componente principal de la calidad de la atención en salud ${ }^{(9)}$, ellos están potencialmente en riesgo de ser afectados por el estrés ${ }^{(10)}$, estado asociado principalmente a un ambiente laboral desagradable, desencadenando lo que se denomina como Burnout (11). No obstante, el estrés podría provenir de otras fuentes, como el ocasionado por situaciones inesperadas de la vida cotidiana. Es probable que residir en Lima Metropolitana, debido a su condición de ciudad capital, sobrepoblada, con una gran cantidad de horas al día invertidas en movilizarse, debido al tránsito saturado y con problemas de inseguridad ciudadana, expongan a los profesionales de salud a situaciones generadoras de estrés. En ese contexto, consideramos que no se ha determinado si el estrés percibido (EP), por situaciones de la vida diaria, podría estar relacionado a la intención de migrar de Lima para trabajar en el interior del país. Por ello, el objetivo del presente estudio fue evaluar la asociación entre el EP y la IMIP en una muestra de médicos y enfermeros que residían en la ciudad de Lima durante el 2015.

\section{MENSAJES CLAVE}

Motivación para realizar el estudio. En Perú existe una carencia de recursos humanos en salud en el interior del país. La migración de los profesionales de salud de Lima Metropolitana a provincias podría estar asociado a la percepción de estrés, debido a situaciones inesperadas e impredecibles que escapan de su control, que podrían afectar su calidad de vida.

Principales hallazgos. La frecuencia de intención de migrar al interior del país en los participantes fue de 4,6\%. Una mayor percepción de estrés se asoció a la intención de migrar al interior del país.

Implicancias. Los hallazgos pueden servir de base para la propuesta de intervenciones de salud informadas en la evidencia.

\section{MATERIALES Y MÉTODOS}

\section{DISEÑO Y ESTUDIO}

El presente estudio fue un análisis secundario de datos, empleando el cuestionario 2 de la Encuesta Nacional de Satisfacción de Usuarios en Salud (ENSUSALUD) 2015. ENSUSALUD es una encuesta que consta de seis cuestionarios aplicados anualmente a usuarios de los servicios de salud para valorar su nivel de satisfacción ${ }^{(12)}$.

El cuestionario 2, evalúa al personal médico y profesionales de enfermería. Este cuestionario fue aplicado en 179 EE.SS. (hospitales, clínicas y centros médicos) del Ministerio de Salud (MINSA), Seguridad Social (EsSalud), Sanidades de las Fuerzas Armadas y Policiales (FFAAPP) y el sector privado de las 25 regiones del Perú.

\section{POBLACIÓN, MUESTRA Y MUESTREO}

La población estuvo constituida por médicos y enfermeros. El tamaño muestral fue calculado en 5067 personas. En el cálculo se utilizó un efecto de diseño de 1,2 y una posibilidad de satisfacción del $30 \%$ de acuerdo con los resultados de la encuesta ENSUSALUD 2014, un nivel de confianza del $95 \%$ y un margen de error entre 3 y 7,5 puntos porcentuales. Debido a que no se definió el tamaño muestral para el presente estudio, se realizó el cálculo de la potencia estadística para la asociación planteada, siendo de $60 \%$. El muestreo fue probabilístico, estratificado y bietápico. Las unidades primarias de muestreo fueron los EE.SS., y las unidades secundarias de muestreo fueron los médicos y enfermeras.

\section{CRITERIOS DE ELEGIBILIDAD}

Se incluyeron en el estudio a aquellos profesionales que únicamente residían en Lima Metropolitana. Para 
seleccionarlos se utilizó la pregunta: ¿en qué departamento, provincia y distrito vive usted? Se excluyeron a aquellos participantes que tuvieron intención de migrar al extranjero o no estaban seguros de su decisión de migrar.

\section{VARIABLES Y MEDICIONES}

La variable de resultado fue la intención de migrar al interior del país (IMIP) por parte de médicos y enfermeras que vivían en Lima Metropolitana. Para determinar dicha variable se realizó las siguientes preguntas: “¿Ud. tiene planes de migrar a otro lugar?" y "¿a qué departamento del Perú o país tiene planificado migrar?". Las respuestas fueron divididas en dos opciones para el primer caso (sí=1 y no=2) y en tres opciones para el segundo caso (departamento=1, país=2 y no lo ha decidido=3). Se consideró como IMIP a aquellos participantes que vivían en Lima Metropolitana y planeaban migrar a algún otro departamento del Perú.

La variable de exposición fue el estrés percibido durante el último mes (previo a la recolección de datos), para lo que se empleó el Perceived stress scale. Esta herramienta validada al idioma español en el año 2006 en una población española consta de 14 preguntas, las cuales poseen un formato de respuesta de una escala de Likert y cuenta con un alfa de Cronbach de 0,86 ${ }^{(13,14)}$. El máximo puntaje posible de obtener en el cuestionario es de 56 puntos. Se interpreta un mayor puntaje como una mayor percepción de estrés por el participante. Esta variable fue evaluada como numérica continua.

Otras variables seleccionadas fueron el sexo, la edad, la profesión (medicina/enfermería), la institución de procedencia (clínicas privadas, MINSA, EsSalud, FFAAPP), pareja estable (se consideró a los participantes casados o convivientes como el estrato de pareja estable), tipo de contrato con el EE.SS., el número de personas que dependen económicamente del encuestado, el contar con alguna especialidad (sí/no), el número de años trabajando en el sector salud, el tipo de trabajo en su EE.SS. (permanente, temporal o estacional), el número de horas semanales de trabajo remuneradas, el nivel de ingreso mensual en soles, la categoría del EE.SS. para el que trabaja y la satisfacción en relación a su trabajo (satisfecho/a, ni satisfecho/a ni insatisfecho/a, insatisfecho/a). Estas variables fueron elegidas en base a estudios previos ${ }^{(7,8)}$.

\section{ASPECTOS ÉTICOS}

ENSUSALUD 2015 es de acceso público en el portal web: http://portales.susalud.gob.pe/web/portal/243. La base de datos no presenta identificadores, con lo cual se garantiza la confidencialidad de la información de los participantes. La recolección de datos fue realizada previo consentimiento verbal, no involucró la toma de muestras biológicas y fue realizada para fines de gestión de los servicios de salud, de esta forma, no se puso en riesgo a ningún participante.

\section{ANÁLISIS ESTADÍSTICO}

Se utilizó el paquete estadístico STATA $®$ v14.0 (Stata Corporation, College Station, Texas, USA). Las variables categóricas fueron presentadas mediante frecuencias y porcentajes. Las variables numéricas se presentaron como medias con desviación estándar o medianas con rangos intercuartílicos, según corresponda. El análisis bivariado entre las variables categóricas se realizó usando chi cuadrado. Se empleó t de Student o suma de rangos de Wilcoxon, para evaluar variables numéricas, según corresponda. Para relacionar el puntaje de EP y variables de tipo ocupacional se utilizó la correlación de Spearman. Se consideró un valor de $p<0,05$ como estadísticamente significativo.

Para valorar la asociación entre el EP y la IMIP, realizamos modelos de regresión logística crudos y ajustados. La medida de asociación reportada fue el odds ratio (OR), con sus respectivos intervalos de confianza al $95 \%$ (IC95\%). Dado que la variable independiente es numérica, se procedió a evaluar el supuesto de linealidad entre el logaritmo de la odds de IMIP y EP usando métodos gráficos y el método del producto cruzado del logaritmo de las variables independientes ${ }^{(15)}$.

Se construyó un modelo global evaluando a la población total del estudio. Para valorar la posible interacción entre el grupo profesional (medicina y enfermería) y el EP se plantearon dos enfoques; en el primero, se realizó un análisis estratificado según el grupo profesional (modelos separados) y en el segundo enfoque se llevó a cabo un modelo de regresión logística que incluyó una variable de interacción entre los grupos profesionales y el EP. Para construir los modelos ajustados se incluyeron como variables confusoras a aquellas cuya asociación con nuestra variable resultado haya sido descrita en la literatura: ingreso económico mensual, estado civil y satisfacción laboral en el modelo que evaluó la asociación entre la IMIP y el estrés percibido en enfermeros ${ }^{(7)}$ e ingreso económico mensual y estado civil en el modelo que evaluó esta asociación en médicos ${ }^{(8)}$.

\section{RESULTADOS}

\section{ASPECTOS GENERALES DE LA POBLACIÓN}

En total, ENSUSALUD 2015 tuvo 5067 encuestados, entre médicos y enfermeros. Se excluyeron a 324 participantes por haber tenido la intención de migrar al extranjero, o no haber decidido al momento de la recolección de datos, si deseaban o no migrar. Asimismo, 3947 personas fueron retiradas por no residir en Lima Metropolitana. Finalmente, se analizaron datos de 796 profesionales (Figura 1). 


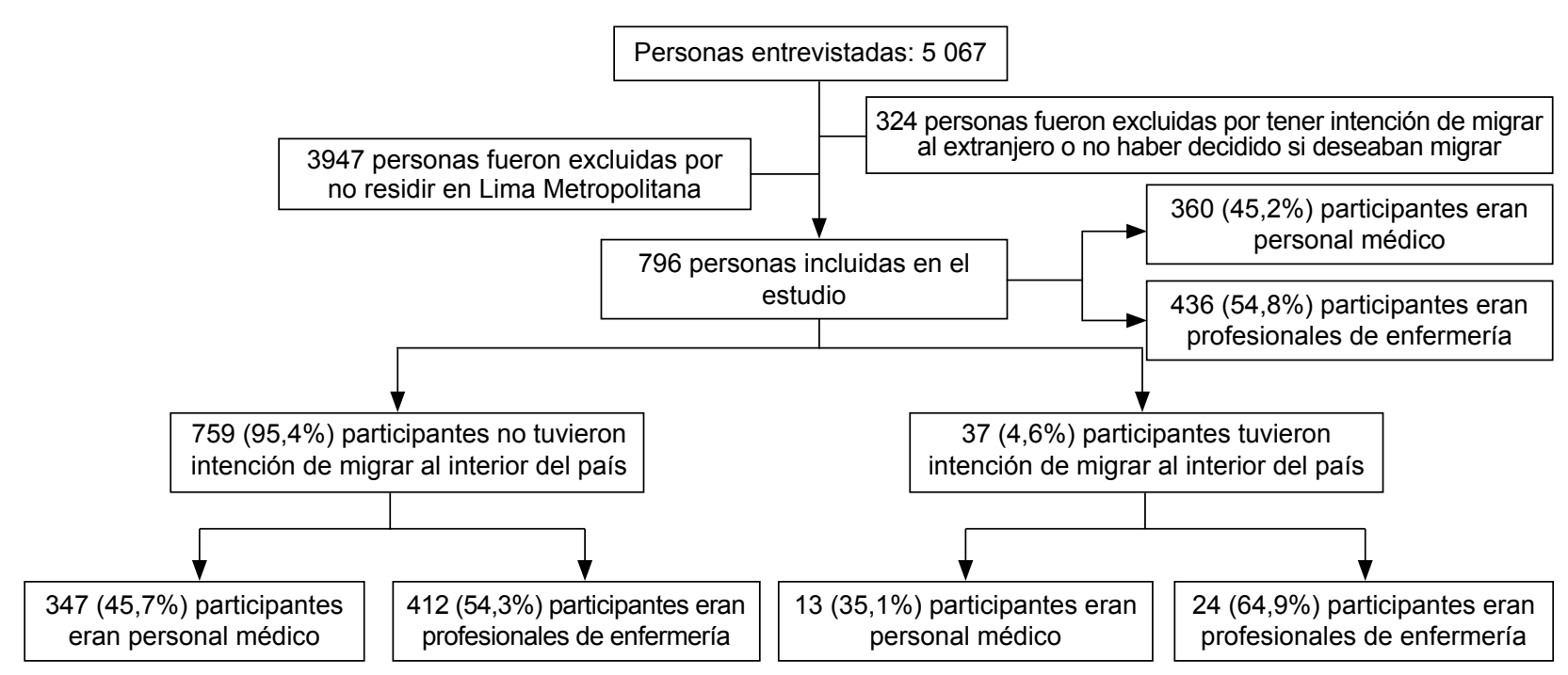

Figura 1. Flujograma de la selección de participantes incluidos en el análisis (ENSUSALUD 2015)

El puntaje promedio de estrés percibido en el total de la población estudiada fue de $20,2 \pm 6,1$. De las 796 personas incluidas en el estudio, $360(45,2 \%)$ eran médicos y $436(54,8 \%)$ eran enfermeros. Asimismo, solo $37(4,6 \%)$ participantes tuvieron IMIP. De estos, 13 $(35,1 \%)$ eran médicos y $24(64,9 \%)$ eran enfermeros (Tabla 1).

\section{ASPECTOS GENERALES DE LOS PROFESIONALES DE ENFERMERÍA}

Se observó que nueve de cada diez participantes eran mujeres; $173(39,7 \%)$ eran menores de 39 años; seis de cada diez contaban con una pareja estable. Además, $236(54,1 \%)$ laboraban en el MINSA y 253 $(58,0 \%)$ contaban con alguna especialidad. Asimismo, $32(7,3 \%)$ laboraban en su EE.SS. de forma temporal o estacional y solo nueve $(2,1 \%)$ percibía un nivel de ingreso mensual mayor a 5000 soles (1572,3 USD de acuerdo al cambio promedio registrado en el año 2015; $1 \mathrm{USD}=3,18$ soles). Adicionalmente, ocho de cada diez participantes laboraban en un EE.SS. de tercer nivel y $93(21,3 \%)$ se sentían insatisfechos con relación a su trabajo (Tabla 1).

\section{ASPECTOS GENERALES DEL PERSONAL MÉDICO}

De los 360 participantes, 237 (65,8\%) eran hombres, $177(49,2 \%)$ fueron mayores de 51 años, tres de cada diez no contaban con una pareja estable y $194(53,9 \%)$ laboraban en el MINSA. Asimismo, 69 (19,2\%) no contaban con alguna especialidad médica, cinco de cada diez percibían un ingreso mensual mayor a 5000 soles (1572,3 USD) y $80(22,2 \%)$ participantes se sentían insatisfechos con relación a su trabajo (Tabla 1).

\section{ANÁLISIS BIVARIADO SEGÚN LA INTENCIÓN DE MIGRAR AL INTERIOR DEL PAÍS}

Con relación a los profesionales de enfermería, se hallaron diferencias estadísticamente significativas únicamente entre la IMIP y el EP. Se encontraron diferencias significativas con relación a la IMIP y las siguientes variables en personal médico: la edad, el contar con alguna especialidad y el tiempo trabajando en el sector salud en años (Tabla 2). Asimismo, el 46,0\% de los participantes con IMIP refirieron su deseo de migrar a la costa, mientras que solo el $10,8 \%$ hizo lo mismo para la selva. Por otro lado, el motivo más influyente en el deseo de migrar de estos profesionales fue el de tipo familiar $(54,1 \%)$.

\section{ESTRÉS PERCIBIDO Y GRUPOS OCUPACIONALES}

El puntaje promedio de EP en enfermeros fue de 20,6 $\pm 5,9$ mientras que en los médicos fue de 19,8 $\pm 6,1$, no siendo la diferencia estadísticamente significativa $(p=0,079)$. El puntaje promedio de EP en enfermeros fue mayor en el grupo con IMIP comparado con quienes no querían migrar $(23,0 \pm 7,2$ vs. $20,4 \pm 5,9 ; p=0,039)$. En contraste, no hubo diferencias significativas en el puntaje promedio de EP entre los médicos con y sin IMIP $(22,6 \pm 5,5$ vs. $19,7 \pm 6,1 ; p=0,096)$.

No se encontró correlación entre el puntaje de EP y variables relacionadas al trabajo como los años de trabajo, el nivel de ingreso y el nivel de complejidad del EE.SS. No obstante, se encontró correlación inversa o negativa entre el puntaje de EP y la satisfacción con el trabajo $(r=-0,28 ; p<0,01)$. 
Tabla 1. Características generales de los médicos y enfermeros incluidos en el estudio ( $N=796)$

\begin{tabular}{|c|c|c|c|c|}
\hline \multirow{2}{*}{ Características } & Total & Enfermeros $(n=436)$ & Médicos $(n=360)$ & \multirow{2}{*}{$\begin{array}{l}\text { Valor } \\
\text { de } p\end{array}$} \\
\hline & n (\%) & $\mathrm{n}(\%)$ & n (\%) & \\
\hline Estrés percibido (puntaje) ${ }^{\star}$ & $20,2 \pm 6,1$ & $20,6 \pm 5,9$ & $19,8 \pm 6,1$ & 0,079 \\
\hline Sexo & & & & $<0,001$ \\
\hline Masculino & $270(33,9)$ & $33(7,6)$ & $237(65,8)$ & \\
\hline Femenino & $526(66,1)$ & $403(92,4)$ & $123(34,2)$ & \\
\hline Edad* $^{*}$ & $45,2 \pm 11,8$ & $42,5 \pm 10,6$ & $48,6 \pm 12,3$ & $<0,001$ \\
\hline$<39$ años & $266(33,4)$ & $173(39,7)$ & $93(25,8)$ & $<0,001$ \\
\hline 39 a 51 años & $251(31,5)$ & $161(36,9)$ & $90(25,0)$ & \\
\hline >51 años & $279(35,1)$ & $102(23,4)$ & $177(49,2)$ & \\
\hline Pareja estable & & & & $<0,001$ \\
\hline Sí & $515(64,7)$ & $254(58,3)$ & $261(72,5)$ & \\
\hline No & $281(35,3)$ & $182(41,7)$ & $99(27,5)$ & \\
\hline Institución de procedencia & & & & 0,832 \\
\hline Clínicas privadas & $128(16,1)$ & $72(16,5)$ & $56(15,6)$ & \\
\hline Ministerio de Salud (MINSA) & $430(54,0)$ & $236(54,1)$ & $194(53,9)$ & \\
\hline Seguridad Social (EsSalud) & $224(28,1)$ & $119(27,3)$ & $105(29,1)$ & \\
\hline Fuerzas Armadas y Policiales & $14(1,8)$ & $9(2,1)$ & $5(1,4)$ & \\
\hline Tipo de contrato con el establecimiento de salud & & & & $<0,001$ \\
\hline Nombrado/permanente & $370(46,5)$ & $194(44,5)$ & $176(48,9)$ & \\
\hline Locación de servicios & $65(8,2)$ & $16(3,7)$ & $49(13,6)$ & \\
\hline Contrato administrativo de servicios & $144(18,1)$ & $110(25,2)$ & $34(9,4)$ & \\
\hline Contrato a plazo fijo & $43(5,4)$ & $25(5,7)$ & $18(5,0)$ & \\
\hline Plazo indeterminado o indefinido & $145(18,2)$ & $77(17,7)$ & $68(18,9)$ & \\
\hline Otros & $29(3,6)$ & $14(3,2)$ & $15(4,2)$ & \\
\hline Personas que dependen económicamente del encuestado* & $1,9 \pm 1,4$ & $1,9 \pm 1,3$ & $2,0 \pm 1,5$ & 0,688 \\
\hline Posee alguna especialidad & & & & $<0,001$ \\
\hline Sí & $544(68,3)$ & $253(58,0)$ & $291(80,8)$ & \\
\hline No & $252(31,7)$ & $183(42,0)$ & $69(19,2)$ & \\
\hline Posee planes de migrar al interior del país & & & & 0,207 \\
\hline No & $759(95,4)$ & $412(94,5)$ & $347(96,4)$ & \\
\hline Sí & $37(4,6)$ & $24(5,5)$ & $13(3,6)$ & \\
\hline Tiempo trabajando en el sector salud en años ${ }^{\dagger}$ & $17(8-26)$ & $14(8-23)$ & $20(10-28)$ & $<0,001$ \\
\hline Tipo de trabajo en su establecimiento de salud & & & & 0,418 \\
\hline Permanente & $733(92,1)$ & $404(92,7)$ & $329(91,4)$ & \\
\hline Temporal o estacional & $63(7,9)$ & $32(7,3)$ & $31(8,6)$ & \\
\hline Horas semanales de trabajo remuneradas ${ }^{\dagger}$ & $40(36-50)$ & $36(36-42,5)$ & $48(38-58)$ & $<0,001$ \\
\hline Ingreso mensual soles / dólares ${ }^{\ddagger}$ & & & & $<0,001$ \\
\hline $\mathrm{S} /<2000 /(628,9$ USD $)$ & $99(12,4)$ & $92(21,1)$ & $7(2,0)$ & \\
\hline S/ 2000-3000 / (628,9 - 943,4 USD) & $159(20,0)$ & $142(32,6)$ & $17(4,6)$ & \\
\hline S/ 3000-5000 / (943,4 - 1572,3 USD) & $361(45,4)$ & $193(44,2)$ & $168(46,7)$ & \\
\hline S/ >5000 / (1572,3 USD) & $177(22,2)$ & $9(2,1)$ & $168(46,7)$ & \\
\hline Nivel de atención & & & & 0,520 \\
\hline I (Primer nivel) & $54(6,8)$ & $26(6,0)$ & $28(7,8)$ & \\
\hline II (Segundo nivel) & $93(11,7)$ & $49(11,2)$ & $44(12,2)$ & \\
\hline III (Tercer nivel) & $649(81,5)$ & $361(82,8)$ & $288(80,0)$ & \\
\hline Satisfacción con relación a su trabajo & & & & 0,949 \\
\hline Satisfecho/a & $486(61,1)$ & $267(61,2)$ & $219(60,8)$ & \\
\hline Ni satisfecho/a ni insatisfecho/a & $137(17,2)$ & $76(17,4)$ & $61(16,9)$ & \\
\hline Insatisfecho/a & $173(21,7)$ & $93(21,4)$ & $80(22,3)$ & \\
\hline
\end{tabular}

* Media \pm desviación estándar,

† Mediana (rango intercuartílico)

‡ tipo de cambio promedio registrado en el año 2015 ; 1 USD=3,18 soles 
Tabla 2. Características de los médicos y enfermeros que residen en Lima Metropolitana de acuerdo a su intención de migrar al interior del país $(\mathrm{N}=796)$

\begin{tabular}{|c|c|c|c|c|c|c|}
\hline \multirow{3}{*}{ Características } & \multicolumn{3}{|c|}{ Enfermeros $(n=436)$} & \multicolumn{3}{|c|}{ Médicos (n=360) } \\
\hline & $\begin{array}{c}\text { Sin intención } \\
\text { de migrar al } \\
\text { interior del } \\
\text { país }(n=412) \\
\end{array}$ & $\begin{array}{c}\text { Con intención } \\
\text { de migrar al } \\
\text { interior del } \\
\text { país }(n=24) \\
\end{array}$ & $\begin{array}{l}\text { Valor } \\
\text { de } p\end{array}$ & $\begin{array}{c}\text { Sin intención } \\
\text { de migrar al } \\
\text { interior del } \\
\text { país }(n=347)\end{array}$ & $\begin{array}{c}\text { Con intención } \\
\text { de migrar al } \\
\text { interior del } \\
\text { país }(n=13) \\
\end{array}$ & $\begin{array}{l}\text { Valor } \\
\text { de } p\end{array}$ \\
\hline & n (\%) & n (\%) & & n (\%) & n (\%) & \\
\hline Estrés percibido (Puntaje) * & $20,4 \pm 5,9$ & $23,0 \pm 7,2$ & 0,039 & $19,7 \pm 6,1$ & $22,6 \pm 5,5$ & 0,096 \\
\hline Sexo & & & 0,083 & & & 0,792 \\
\hline Masculino & $29(87,9)$ & $4(12,1)$ & & $228(96,2)$ & $9(3,8)$ & \\
\hline Femenino & $383(95,0)$ & $20(5,0)$ & & $119(96,8)$ & $4(3,2)$ & \\
\hline Edad * & $42,7 \pm 10,7$ & $39,6 \pm 7,9$ & 0,169 & $49,0 \pm 12,2$ & $36,7 \pm 9,8$ & $<0,001$ \\
\hline$<39$ años & $160(92,5)$ & $13(7,5)$ & 0,257 & $84(90,3)$ & $9(9,7)$ & 0,001 \\
\hline 39 a 51 años & $153(95,0)$ & $8(5,0)$ & & $88(97,8)$ & $2(2,2)$ & \\
\hline >51 años & $99(97,1)$ & $3(2,9)$ & & $175(98,9)$ & $2(1,1)$ & \\
\hline Pareja estable & & & 0,399 & & & 0,125 \\
\hline Sí & $242(95,3)$ & $12(4,7)$ & & $254(97,3)$ & $7(2,7)$ & \\
\hline No & $170(93,4)$ & $12(6,6)$ & & $93(93,9)$ & $6(6,1)$ & \\
\hline Institución & & & 0,299 & & & 0,409 \\
\hline Clínicas privadas & $71(98,6)$ & $1(1,4)$ & & $56(100,0)$ & $0(0,0)$ & \\
\hline Ministerio de Salud (MINSA) & $220(93,2)$ & $16(6,8)$ & & $185(95,4)$ & $9(4,6)$ & \\
\hline Seguridad Social (EsSalud) & $113(95,0)$ & $6(5,0)$ & & $101(96,2)$ & $4(3,8)$ & \\
\hline Fuerzas Armadas y Policiales & $8(88,9)$ & $1(11,1)$ & & $5(100,0)$ & $0(0,0)$ & \\
\hline Tipo de contrato & & & 0,051 & & & 0,951 \\
\hline Nombrado, permanente & $187(96,4)$ & $7(3,6)$ & & $170(96,6)$ & $6(3,4)$ & \\
\hline Locación de servicios & $16(100,0)$ & $0(0,0)$ & & $47(95,9)$ & $2(4,1)$ & \\
\hline Contrato administrativo de servicios & $97(88,2)$ & $13(11,8)$ & & $33(97,1)$ & $1(2,9)$ & \\
\hline Contrato a plazo fijo & $25(100,0)$ & $0(0,0)$ & & $17(94,4)$ & $1(5,6)$ & \\
\hline Plazo indeterminado o indefinido & $74(96,1)$ & $3(3,9)$ & & $65(95,6)$ & $3(4,4)$ & \\
\hline Otros & $13(92,9)$ & $1(7,1)$ & & $15(100,0)$ & $0(0,0)$ & \\
\hline Personas que dependen del encuestado* & $1,9 \pm 1,3$ & $1,5 \pm 1,3$ & 0,138 & $1,9 \pm 1,4$ & $2,2 \pm 2,7$ & 0,614 \\
\hline Posee alguna especialidad & & & 0,213 & & & 0,005 \\
\hline Sí & $242(95,7)$ & $11(4,3)$ & & $285(97,9)$ & $6(2,1)$ & \\
\hline No & $170(92,9)$ & $13(7,1)$ & & $62(89,9)$ & $7(10,1)$ & \\
\hline Tiempo trabajando en el sector salud en años ${ }^{\dagger}$ & $14(8-23,5)$ & $10(6-15)$ & 0,067 & $21(11-28)$ & $8(4-15)$ & 0,002 \\
\hline Tipo de trabajo en su establecimiento de salud & & & 0,848 & & & 0,904 \\
\hline Permanente & $382(94,6)$ & $22(5,4)$ & & $317(96,4)$ & $12(3,6)$ & \\
\hline Temporal o estacional & $30(93,8)$ & $2(6,2)$ & & $30(96,8)$ & $1(3,2)$ & \\
\hline Horas semanales de trabajo remuneradas $^{\dagger}$ & $36(36-43)$ & $38(36-38)$ & 0,827 & $48(38-60)$ & $45(43-48)$ & 0,681 \\
\hline Ingreso mensual soles / dólares ${ }^{\ddagger}$ & & & 0,417 & & & 0,309 \\
\hline $\mathrm{S} /<2000$ / (628,9 USD) & $88(95,7)$ & $4(4,3)$ & & $7(100,0)$ & $0(0,0)$ & \\
\hline S/ 2000-3000 / (628,9 - 943,4 USD) & $131(92,3)$ & $11(7,7)$ & & $16(94,1)$ & $1(5,9)$ & \\
\hline S/ 3000-5000 / (943,4 - 1572,3 USD) & $185(95,9)$ & $8(4,1)$ & & $159(94,6)$ & $9(5,4)$ & \\
\hline $\mathrm{S} />5000 /(1572,3$ USD $)$ & $8(88,9)$ & $1(11,1)$ & & $165(98,2)$ & $3(1,8)$ & \\
\hline Nivel de atención & & & 0,470 & & & 0,246 \\
\hline I (Primer nivel) & $25(96,2)$ & $1(3,8)$ & & $28(100,0)$ & $0(0,0)$ & \\
\hline II (Segundo nivel) & $48(98,0)$ & $1(2,0)$ & & $44(100,0)$ & $0(0,0)$ & \\
\hline III (Tercer nivel) & $339(93,9)$ & $22(6,1)$ & & $275(95,5)$ & $13(4,5)$ & \\
\hline Satisfacción con relación a su trabajo & & & 0,069 & & & 0,167 \\
\hline Satisfecho/a & $257(96,3)$ & $10(3,7)$ & & $214(97,7)$ & $5(2,3)$ & \\
\hline Ni satisfecho/a ni insatisfecho/a & $68(89,5)$ & $8(10,5)$ & & $57(93,4)$ & $4(6,6)$ & \\
\hline Insatisfecho/a & $87(93,6)$ & $6(6,4)$ & & $76(95,0)$ & $4(5,0)$ & \\
\hline
\end{tabular}

* media \pm desviación estándar

† mediana (rango intercuartílico)

₹ tipo de cambio promedio registrado en el año $2015 ; 1$ USD $=3,18$ soles 
En cuanto a los puntajes promedio por cada pregunta de la escala de EP empleada, se hallaron diferencias significativas en la primera, segunda y undécima pregunta en el grupo de enfermeros según su IMIP. Por otro lado, se halló diferencias significativas en la primera, segunda, octava y undécima pregunta en el grupo de médicos según su IMIP (Tabla 3).

\section{ANÁLISIS DE REGRESIÓN LOGÍSTICA}

Se halló asociación significativa en el análisis crudo de regresión logística para valorar la asociación entre el puntaje de EP y la IMIP (OR=1,31; IC95\%:1,08-1,60). Tras ajustar por variables confusoras descritas en la literatura y disponibles en la base, la asociación permaneció significativa (OR=1,31; IC95\%:1,07-1,60) (Tabla 4).

El modelo con interacción entre puntaje de estrés y grupo ocupacional ajustado por variables confusoras descritas en la literatura y disponibles en la base, que incluyó a todos los participantes, no evidenció asociación estadísticamente significativa para la asociación entre el puntaje de EP y la IMIP (OR=1,25; IC95\%:0,91-1,72); el valor $p$ de interacción no fue significativo $(p=0,726)$.

En los modelos estratificados por grupo ocupacional, para el grupo de enfermería se halló asociación significativa en el análisis crudo de regresión logística (OR=1,31; IC95\%: 0,95-1,81), así como, tras ajustar por variables confusoras descritas en la literatura y disponibles en la base, la asociación permaneció con significancia estadística (OR=1,30; IC95\%:1,01-1,67).

Asu vez, para el grupo de médicos, no se halló asociación significativa en el análisis crudo (OR=1,31; IC95\%:0,95$1,81)$, ni en el análisis ajustado de regresión logística (OR=1,28; IC95\%:0,89-1,83).

\section{DISCUSIÓN}

La frecuencia de IMIP fue de 4,6\%. Esto es consistente con lo reportado por Mayta-Tristán et al.(8), quienes hallaron que de 782 médicos solo el $7 \%$ tuvo intención de laborar en el interior del país dentro de los próximos cinco años. Sin embargo, ese estudio se llevó a cabo en médicos recién colegiados de Lima, lo que difiere de nuestra investigación, debido a que evaluamos médicos y enfermeras con distintos tiempos de práctica profesional. En ese sentido, no hallamos otros antecedentes locales que hayan evaluado la IMIP, en personal médico y de enfermería en ejercicio profesional, por lo cual resaltamos la importancia de la presente investigación. En adición, cuatro estudios llevados a cabo a nivel local en estudiantes de ciencias de la salud e internos de medicina reportaron frecuencias de intención de trabajar en provincias y en el interior del país de 15\%, 10,9\%, 21,9\% y 5,3\%, respectivamente ${ }^{(16-19)}$. Las posibles explicaciones a las diferencias halladas con los antecedentes podrían deberse a que el personal sanitario evaluado en la presente investigación contaba probablemente con una mayor estabilidad laboral y proyectos de vida ya establecidos, a diferencia del personal de salud recién egresado que podría tener una mayor incertidumbre laboral y proyectos profesionales incipientes, tomando la posibilidad de migrar al interior del país como una opción laboral a futuro.

A nivel internacional existen reportes que exploran las relaciones entre los factores de atracción y retención de personal médico en áreas rurales remotas. En Tailandia existe una mejora considerable del acceso equitativo a la asistencia sanitaria, la estrategia se basa en el reclutamiento de profesionales de la salud del ámbito rural, brindándoles oportunidades de capacitación y la subvención económica durante su formación a cambio de trabajar en áreas remotas después de la graduación ${ }^{(20)}$. En Indonesia, los médicos que trabajan en áreas remotas perciben el doble del salario que sus colegas que trabajan en el ámbito urbano y poseen mayores opciones de acceder a entrenamientos subsidiados por el gobierno (21). En África se han adoptado medidas para la apertura de escuelas de Medicina con un enfoque basado en la atención a la comunidad; específicamente en países como Etiopía, Ghana y Kenia ${ }^{(22)}$.

El Estado Peruano ha intentado descentralizar los RR.HH. en salud, mediante programas como el Servicio Rural y Urbano Marginal de Salud (SERUMS); sin embargo, un estudio muestra que el interés del personal médico por laborar en provincias disminuyó en un $20 \%$ después del SERUMS ${ }^{23)}$. Un incentivo para retener a los RR.HH., podría ser el ingreso directo a programas de residencia en especialidades médicas prioritarias de la región donde se realiza el SERUMS. Otra política de estado, que busca mejorar la gestión de los RR.HH., ha sido la implementación en el año 2013, del Observatorio Nacional de Recursos Humanos en Salud (24); este proporciona información, sobre la disponibilidad regional de RR.HH., para un planeamiento de los servicios de salud y toma de decisiones basada en la información. Asimismo, durante el año 2013 se propuso el incremento de beneficios monetarios y no monetarios para los profesionales de salud en zonas alejadas ${ }^{(24)}$. Los beneficios no monetarios incluyeron mejores condiciones de vivienda, alimentación, becas de capacitación en telemedicina y bonificaciones para el ingreso a una segunda especialización. Sin embargo, a la fecha, este programa de incentivos aún se encuentra en implementación. Por otro lado, consideramos que dada la situación fragmentada del sistema de salud peruano ${ }^{(25)}$, las políticas públicas tendrían un mayor impacto si se articularan en un único esfuerzo, coordinado entre todos los subsistemas de salud existentes en el Perú. 
Tabla 3. Puntajes por cada pregunta del Perceived stress scale según la intención de migrar al interior del país y la profesión $(\mathrm{N}=796)$

\begin{tabular}{|c|c|c|c|c|c|c|}
\hline \multirow{3}{*}{ Preguntas } & \multicolumn{3}{|c|}{ Enfermeros $(n=436)$} & \multicolumn{3}{|c|}{ Médicos $(n=360)$} \\
\hline & $\begin{array}{l}\text { Sin intención } \\
\text { de migrar al } \\
\text { interior del país } \\
(n=412)\end{array}$ & $\begin{array}{l}\text { Con intención } \\
\text { de migrar al } \\
\text { interior del país } \\
(n=24)\end{array}$ & $\begin{array}{l}\text { Valor } \\
\text { de } p\end{array}$ & $\begin{array}{l}\text { Sin intención } \\
\text { de migrar al } \\
\text { interior del país } \\
(n=347)\end{array}$ & $\begin{array}{l}\text { Con intención } \\
\text { de migrar al } \\
\text { interior del país } \\
(n=13)\end{array}$ & $\begin{array}{l}\text { Valor } \\
\text { de } p\end{array}$ \\
\hline & Media \pm DE & Media $\pm \mathrm{DE}$ & & Media \pm DE & Media \pm DE & \\
\hline $\begin{array}{l}\text { En el último mes, ¿con qué } \\
\text { frecuencia ha estado afectado } \\
\text { por algo que ha ocurrido } \\
\text { inesperadamente? }\end{array}$ & $1,7 \pm 1,1$ & $2,1 \pm 1,2$ & 0,035 & $1,7 \pm 1,0$ & $2,5 \pm 1,0$ & 0,002 \\
\hline $\begin{array}{l}\text { En el último mes, ¿con qué } \\
\text { frecuencia se ha sentido } \\
\text { incapaz de controlar las cosas } \\
\text { importantes en su vida? }\end{array}$ & $1,3 \pm 1,0$ & $1,8 \pm 1,3$ & 0,019 & $1,2 \pm 0,8$ & $1,8 \pm 1,3$ & 0,005 \\
\hline $\begin{array}{l}\text { En el último mes, ¿con qué } \\
\text { frecuencia se ha sentido } \\
\text { nervioso o estresado? }\end{array}$ & $1,8 \pm 0,9$ & $2,0 \pm 1,1$ & 0,326 & $1,8 \pm 0,9$ & $2,1 \pm 0,9$ & 0,194 \\
\hline $\begin{array}{l}\text { En el último mes, ¿con qué } \\
\text { frecuencia ha manejado con } \\
\text { éxito los pequeños problemas } \\
\text { irritantes de la vida? }\end{array}$ & $1,2 \pm 1,0$ & $1,4 \pm 1,0$ & 0,517 & $1,0 \pm 0,9$ & $1,1 \pm 1,0$ & 0,853 \\
\hline $\begin{array}{l}\text { En el último mes, ¿con qué } \\
\text { frecuencia ha sentido que ha } \\
\text { afrontado efectivamente los } \\
\text { cambios importantes que han } \\
\text { estado ocurriendo en su vida? }\end{array}$ & $1,1 \pm 0,9$ & $1,3 \pm 1,0$ & 0,438 & $1,0 \pm 0,8$ & $0,9 \pm 0,8$ & 0,474 \\
\hline $\begin{array}{l}\text { En el último mes, ¿con qué } \\
\text { frecuencia ha estado seguro } \\
\text { sobre su capacidad para manejar } \\
\text { sus problemas personales? }\end{array}$ & $1,0 \pm 1,0$ & $1,2 \pm 0,8$ & 0,302 & $0,9 \pm 0,9$ & $0,7 \pm 0,8$ & 0,322 \\
\hline $\begin{array}{l}\text { En el último mes, ¿con qué } \\
\text { frecuencia ha sentido que las } \\
\text { cosas le van bien? }\end{array}$ & $1,0 \pm 0,9$ & $1,0 \pm 0,8$ & 0,736 & $1,0 \pm 0,9$ & $0,9 \pm 0,9$ & 0,697 \\
\hline $\begin{array}{l}\text { En el último mes, ¿con qué } \\
\text { frecuencia ha sentido que no } \\
\text { podía afrontar todas las cosas } \\
\text { que tenía que hacer? }\end{array}$ & $1,6 \pm 1,0$ & $1,9 \pm 1,2$ & 0,131 & $1,5 \pm 0,9$ & $2,0 \pm 1,3$ & 0,041 \\
\hline $\begin{array}{l}\text { En el último mes, ¿con qué } \\
\text { frecuencia ha podido controlar } \\
\text { las dificultades de su vida? }\end{array}$ & $1,0 \pm 0,9$ & $0,9 \pm 0,8$ & 0,641 & $1,0 \pm 0,9$ & $0,9 \pm 0,9$ & 0,800 \\
\hline $\begin{array}{l}\text { En el último mes, ¿con qué } \\
\text { frecuencia se ha sentido que } \\
\text { tenía todo bajo control? }\end{array}$ & $1,1 \pm 0,9$ & $1,2 \pm 0,9$ & 0,690 & $1,1 \pm 0,9$ & $1,1 \pm 0,9$ & 0,941 \\
\hline $\begin{array}{l}\text { En el último mes, ¿con qué } \\
\text { frecuencia ha estado enfadado } \\
\text { porque las cosas que le han } \\
\text { ocurrido estaban fuera de su } \\
\text { control? }\end{array}$ & $1,7 \pm 0,9$ & $2,1 \pm 1,1$ & 0,018 & $1,6 \pm 0,8$ & $2,2 \pm 1,1$ & 0,031 \\
\hline $\begin{array}{l}\text { En el último mes, ¿con qué } \\
\text { frecuencia ha pensado sobre las } \\
\text { cosas que le quedan por hacer? }\end{array}$ & $2,5 \pm 1,0$ & $2,7 \pm 0,8$ & 0,547 & $2,6 \pm 0,9$ & $3,0 \pm 0,9$ & 0,118 \\
\hline $\begin{array}{l}\text { En el último mes, ¿con qué } \\
\text { frecuencia ha podido controlar la } \\
\text { forma de pasar el tiempo? }\end{array}$ & $1,4 \pm 1,0$ & $1,3 \pm 1,0$ & 0,817 & $1,3 \pm 0,9$ & $1,1 \pm 1,0$ & 0,392 \\
\hline $\begin{array}{l}\text { En el último mes, ¿con qué } \\
\text { frecuencia ha sentido que las } \\
\text { dificultades se acumulan tanto } \\
\text { que no puede superarlas? }\end{array}$ & $2,0 \pm 1,3$ & $2,1 \pm 1,2$ & 0,824 & $2,0 \pm 1,3$ & $2,3 \pm 1,3$ & 0,436 \\
\hline
\end{tabular}

DE: Desviación estándar 
Tabla 4. Modelos de regresión logística para evaluar la asociación entre el estrés percibido y la intención de migrar al interior del país según profesión $(\mathrm{N}=796)$

\begin{tabular}{|c|c|c|c|c|c|}
\hline Profesión & Exposición & $\begin{array}{l}\text { Modelo crudo } \\
\text { OR (IC95\%) }\end{array}$ & Valor de $p$ & $\begin{array}{c}\text { Modelo ajustado } \\
\text { OR (IC95\%) }\end{array}$ & Valor de $p$ \\
\hline Global & Estrés percibido & $1,31(1,08-1,60)$ & 0,007 & $1,31(1,07-1,60)^{\dagger}$ & 0,009 \\
\hline Global + interacción & Estrés percibido & $1,18(0,88-1,57)$ & 0,261 & $1,25(0,91-1,72)^{\dagger \S}$ & 0,167 \\
\hline Médicos & Estrés percibido & $1,31(0,95-1,81)$ & 0,099 & $1,28(0,89-1,83)^{\dagger}$ & 0,183 \\
\hline Enfermeros & Estrés percibido & $1,31(1,01-1,69)$ & 0,040 & $1,30(1,01-1,67) \ddagger$ & 0,046 \\
\hline
\end{tabular}

† Ajustado por ingreso económico mensual y estado civil.

‡Ajustado por ingreso económico mensual, estado civil y satisfacción laboral.

$\S$ Valor $p$ de interacción $=0,726$

El presente estudio halló asociación positiva entre el EP y la IMIP en los profesionales de salud encuestados. El EP como constructo medido en la presente investigación, está íntimamente relacionado a la forma en la que las personas reaccionan frente a situaciones inesperadas e impredecibles que escapan de su control. Lima, como ciudad capital del Perú, presenta situaciones cotidianas que son potenciales estresores, como la delincuencia e inseguridad ciudadana, la contaminación ambiental y sonora, la tugurización del transporte público unida al tráfico saturado, deriva en falta de tiempo para momentos de ocio, en desmedro de la calidad de vida ${ }^{(26)}$. Estos estresores podrían motivar a los profesionales de la salud a buscar ámbitos donde las situaciones que escapan de su control tengan una menor repercusión sobre su calidad de vida. Asimismo, los profesionales sanitarios podrían percibir mayor estrés en caso deseen migrar al interior del país, pero no puedan concretarlo.

Por otro lado, de manera exploratoria, los hallazgos del estudio no muestran una modificación de efecto debido al grupo profesional (medicina o enfermería) en la IMIP. Se exploró la interacción, dado que las características sociodemográficas varían entre profesionales de salud, así como el tipo de estresores a los que los expone su labor asistencial, como se revela en estudios relacionados al estrés ocupacional ${ }^{(27,28)}$.

Nuestros hallazgos podrían servir como insumo en la toma de decisiones de los gestores de RR.HH. en salud, para diseñar y ejecutar intervenciones que permitan una distribución equitativa de los recursos al interior del país. Todo ello está alineado con la actual política del gobierno peruano, liderada por la Superintendencia Nacional de Salud (SUSALUD), que intenta empoderar a los ciudadanos respecto del uso de sus derechos en salud ${ }^{(29)}$. De poder concretar intervenciones o políticas públicas con éxito, la distribución equitativa de los RR.HH. en salud al interior del país permitiría un goce adecuado del derecho a la salud.
El presente estudio tuvo limitaciones: 1) Al ser un análisis secundario de datos, estos no fueron concebidos para responder a nuestra pregunta de investigación, por lo cual, podría haber otras variables que explicarían esta asociación y que hubiese sido relevante incluir, como: tener familiares en el interior del país, radicar fuera de Lima, o ser quechua hablante. 2) El diseño transversal, no permitió evaluar asociación causal entre el EP y la IMIP, no obstante, consideramos que el presente análisis representa una primera aproximación a esta problemática en el Perú. Asimismo, no permite controlar la posibilidad de causalidad reversa entre nuestras dos variables de interés. 3) No fue posible determinar si los participantes con IMIP van a poder concretarlo durante los meses o años siguientes.

En conclusión, la IMIP se presentó en uno de cada 20 profesionales de la salud, el tener una mayor percepción de estrés resultó ser un factor asociado a la IMIP. Se recomienda llevar a cabo estudios de seguimiento que corroboren nuestros hallazgos, valorar el EP y analizar otras exposiciones plausibles de ser modificadas como factores causales de la IMIP, y valorar si influyen en la decisión final, de migrar o no, del profesional de salud peruano.

Contribuciones de autoría: BSU, SVV, DUP y VBZ participaron en el diseño del estudio y la concepción del artículo. VBZ y DUP contribuyeron con la adquisición de datos. VBZ y DUP realizaron la interpretación de los datos. BSU, SVV y DUP realizaron la escritura del borrador del artículo. Todos los autores hicieron la revisión crítica del artículo, aprobaron la versión final y se hacen responsables del contenido.

Tipo de financiamiento: ENSUSALUD fue financiado por la Superintendencia Nacional de Salud (SUSALUD) sin embargo, el presente trabajo ha sido autofinanciado por los autores.

Conflictos de interés: los autores declaran no tener conflictos de interés con respecto a la publicación del presente artículo. 


\section{REFERENCIAS BIBLIOGRÁFICAS}

1. Dirección General de Gestión del Desarrollo de Recursos Humanos. Segunda medición de las metas regionales de recursos humanos para la salud, Perú 2007-2015. Lima: MINSA;2013. (citado el 15 de enero de 2017). Disponible en: http://www.minsa.gob.pe/dggdrh/ libros/pdf/s1/I-15.\%20Segunda\%20 Medicion.pdf

2. Instituto Nacional de Estadística e Informática. Informe técnico: Evolución de la pobreza monetaria 2009-2015: Lima: INEI; 2016. (citado el 15 de enero de 2017). Disponible en: https://www. inei.gob.pe/media/MenuRecursivo/ publicaciones_digitales/Est/Lib1347/ libro.pdf

3. Ramirez J, Rivera P, Becerra C, Pena S, Arteaga Ó. Evaluación de la experiencia de participantes en estrategia de dotación de médicos generales en zonas rurales. Rev Med Chil. 2016;144(4):426-33. doi: 10.4067/S0034-98872016000400002

4. Carrasco Cortez V, Lozano Salazar E, Velásquez Pancca E. Análisis actual y prospectivo de la oferta y demanda de médicos en el Perú 2005-2011. Acta méd peru. 2008;25(1):22-9.

5. Mayta-Tristán P, Cuentas $M$, NúñezVergara M. Responsabilidad de las instituciones ante la proliferación de escuelas de medicina en el Perú. Acta méd peru. 2016;33(3):178-82.

6. Gamero Requena J. Reclutamiento y migración de recursos humanos de la salud. Informe de consultoría. Lima: Organización Panamericana de la Salud; 2010. (citado el 10 de enero de 2017). Disponible en: https://goo.gl/9jCdMo

7. SiantzdeLeon ML, MalvárezS. Migration of Nurses: A Latin American Perspective. Online J Issues Nurs. 2008;13(2). doi: 10.3912/OJIN.Vol13No02Man02.

8. Mayta-Tristán P, Mejia CR, Riega-Lopez P, Rojas-Mezarina L, Posso M, MezonesHolguín E. Proyección de trabajo en el interior del país y factores asociados en médicos recién colegiados de Lima, Perú 2010. Rev Peru Med Exp Salud Publica. 2011;28(2):186-93.

9. Datos del Observatorio mundial de la salud [Internet]. Ginebra: Organización Mundial de la Salud; 2017. (citado el 15 de enero de 2017). Disponible en: http:// www.who.int/gho/es/

10. Squires A, Finlayson C, Gerchow L, Cimiotti JP, Matthews A, Schwendimann $\mathrm{R}$, et al. Methodological considerations when translating "burnout". Burn Res. 2014;1(2):59-68.

11. Pulcrano M, Evans SR, Sosin M. Quality of life and burnout rates across surgical specialties: a systematic review. JAMA Surg. 2016 Oct 1;151(10):970-978. doi: 10.1001/jamasurg.2016.1647.

12. Mezones-Holguín E, Solis-Cóndor R, Benites-Zapata VA, Garnica-Pinazo G, Marquez-Bobadilla E, TantaleánDel-Águila $\mathrm{M}$, et al. Diferencias institucionales en el insuficiente acceso efectivo a medicamentos prescritos en instituciones prestadoras de servicios de salud en Perú: Análisis de la Encuesta Nacional de Satisfacción de Usuarios de los Servicios de Salud (ENSUSALUD 2014). Rev Peru Med Exp Salud Publica. 2016;33(2):205-14.

13. Remor E. Psychometric properties of a European Spanish version of the Perceived Stress Scale (PSS). Span J Psychol. 2006;9(1):86-93.

14. Cohen S, Kamarck T, Mermelstein R. A global measure of perceived stress. J Health Soc Behav. 1983;24(4):385-96.

15. Hosmer DW, Lemeshow S, Rodney XS. Applied logistic regression: 3rd Edition. Toronto: John Wiley \& Sons; 2013.

16. Ramírez Huaranga MA. Expectativas de los internos de medicina humana de 5 hospitales generales de Lima y Callao acerca de su ejercicio profesional y su inclinación por la atención primaria de salud: Lima-2006 [Tesis]. Universidad Nacional Mayor de San Marcos, 2008. (citado el 10 de enero de 2017). Disponible en: http://cybertesis.unmsm. edu.pe/handle/cybertesis/3048.

17. Portal Cavero KA. Expectativas del ejercicio medico profesional en los internos de medicina del Hospital Nacional Arzobispo Loayza, 2015. [Tesis]. Universidad Nacional Mayor de San Marcos, 2015. (citado el 10 de enero de 2017). Disponible en: http://cybertesis. unmsm.edu.pe/handle/cybertesis/4134.

18. Inga Berrospi F. Asociación entre las expectativas de trabajo en atención primaria en salud y la intención de migración externa en estudiantes de medicina de la Universidad de San Martín de Porres. 2013. [Tesis]. Universidad de San Martín de Porres, 2013. (citado el 5 de enero de 2017). Disponible en: http:// www.repositorioacademico.usmp.edu. pe/handle/usmp/1373.

19. Tarqui-Mamani CB, Sanabria Rojas HA, Zárate Cárdenas E. Expectativas de laborar en el primer nivel de atención de salud de los estudiantes de una facultad de medicina de Lima, Perú. An Fac med. 2015;76(1):57-62. doi:10.15381/anales. v76i1.11076

20. Wibulpolprasert S, Pengpaibon P. Integrated strategies to tackle the inequitable distribution of doctors in Thailand: four decades of experience. Hum Resour Health. 2003;1(1):12.

21. Chomitz KM, Setiadi G, Azwar A, Widiyarti N. What do doctors want? Developing incentives for doctors to serve in Indonesias rural and remote areas. Policy research working paper 1888 . Washington, DC: World Bank; 1998.

22. Dovlo D. The brain drain and retention of health professionals in Africa. Case studies prepared for regional training conference: Improving tertiary education in sub-Saharan Africa: things that work! Accra, 23-25 September 2003.

23. Mejía CR, Quiñones-Laveriano DM. SERUMS y la migración de médicos: a propósito de una cohorte de médicos de Lima. Rev Peru Med Exp Salud Publica. 2015;32(2):405-6.

24. Perú MdS. Lineamientos y medidas de reforma del sector salud. MINSA Lima; 2013.

25. Alcalde-Rabanal JE, Lazo-González O, Nigenda G. Sistema de salud de Perú. Salud Publica Mex. 2011;53 Suppl 2:s243-54

26. Encuesta Lima Cómo Vamos 2012. Informe de Percepción sobre Calidad de Vida. Lima: Lima Cómo Vamos; 2012. (citado el 15 de enero de 2017). Disponible en: http://www.limacomovamos. org/cm/wp-content/uploads/2012/01/ EncuestaLimaComoVamos-2011.pdf--*

27. Gundersen L. Physician burnout. Ann Intern Med. 2001;135(2):145-8.

28. McVicar A. Workplace stress in nursing: a literature review. J Adv Nurs. 2003;44(6):633-42.

29. Mezones-Holguín E, Díaz-Romero $\mathrm{R}$, Castillo-Jayme J, Jerí-de-Pinho $\mathrm{M}$, Benites-Zapata V, Marquez-Bobadilla E, et al. Promoción de los derechos en salud en Perú: una aproximación desde la perspectiva de acción de la Superintendencia Nacional de Salud. Rev Peru Med Exp Salud Publica. 2016;33(3):520-528. doi: 10.17843/ rpmesp.2016.333.2302.

Correspondencia: Vicente A Benites-Zapata Dirección: Centro de Investigación en Epidemiología Clinica y Medicina Basada en Evidencias, Instituto de Investigación, Facultad de Medicina Humana, Universidad de San Martin de Porres (USMP), Campus Avenida Alameda del Corregidor 1531, La Molina. Lima, Perú

Teléfono: (+51) 995523081

Correo:vbeniteszapata@gmail.com 